Development of a Time-resolved Soft X-ray Spectrometer for Laser Produced Plasma Experiments

K. V. Cone, J. Dunn, M. B. Schneider, H. A. Baldis, G. V. Brown, J. Emig, D. L. James, M. J. May, J. Park, R. Shepherd, K. Widmann

May 13,2010

High Temperature Plasma Diagnostics 2010 Wildwood, NJ, United States May 16, 2010 through May 20, 2010 
This document was prepared as an account of work sponsored by an agency of the United States government. Neither the United States government nor Lawrence Livermore National Security, LLC, nor any of their employees makes any warranty, expressed or implied, or assumes any legal liability or responsibility for the accuracy, completeness, or usefulness of any information, apparatus, product, or process disclosed, or represents that its use would not infringe privately owned rights. Reference herein to any specific commercial product, process, or service by trade name, trademark, manufacturer, or otherwise does not necessarily constitute or imply its endorsement, recommendation, or favoring by the United States government or Lawrence Livermore National Security, LLC. The views and opinions of authors expressed herein do not necessarily state or reflect those of the United States government or Lawrence Livermore National Security, LLC, and shall not be used for advertising or product endorsement purposes. 


\title{
Development of a Time-resolved Soft X-ray Spectrometer for Laser Produced Plasma Experiments ${ }^{a)}$
}

\author{
K. V. Cone ${ }^{1,2, b)}$ J. Dunn, ${ }^{1}$ M. B. Schneider, ${ }^{1}$ H. A. Baldis, ${ }^{2}$ G. V. Brown, ${ }^{1}$ J. Emig, ${ }^{1}$ \\ D. L. James, ${ }^{1}$ M. J. May, ${ }^{1}$ J. Park, ${ }^{1,2}$ R. Shepherd ${ }^{1}$, and K. Widmann ${ }^{1}$ \\ ${ }^{1}$ Lawrence Livermore National Laboratory, Livermore, CA 94551, USA \\ ${ }^{2}$ University of California at Davis, Davis, CA 95616, USA
}

(Presented XXXXX; received XXXXX; accepted XXXXX; published online XXXXX)

\begin{abstract}
A 2400 line/mm variable spaced grating spectrometer (VSG) has been used to measure soft x-ray emission (8 - $22 \AA)$ from laser-produced plasma experiments at Lawrence Livermore National Laboratory's Compact Multipulse Terrawatt (COMET) Laser Facility. The spectrometer was coupled to a Kentech x-ray streak camera to study the temporal evolution of soft x-rays emitted from the back of mylar and copper foils irradiated at $10^{15} \mathrm{~W} / \mathrm{cm}^{2}$. The instrument demonstrated a resolving power of $\sim 120$ at $19 \AA$ with a time resolution of 31 ps. The time-resolved copper emission spectrum was consistent with a photodiode monitoring the laser temporal pulse shape and indicated that the soft x-ray emission follows the laser heating of the target. The time and spectral resolution of this diagnostic make it useful for studies of high temperature plasmas.
\end{abstract}

\section{INTRODUCTION}

X-ray spectroscopy is important in the study of high temperature plasmas, such as those found in astrophysical objects and in short-lived systems generated through laser-matter interactions in the laboratory. X-ray spectra can be used to determine various characteristics of plasmas including temperature and density ${ }^{1}$ without directly perturbing the system.

X-ray spectroscopy is used in current experiments to diagnose the heating mechanisms of a short pulse laser interacting with near-solid density targets. For this study, a grating spectrometer sensitive to soft $\mathrm{x}$-rays was developed at Lawrence Livermore National Laboratory (LLNL). It was designed to measure the L-shell emission of radiatively-heated materials $^{2}$ to diagnose their temperature. Recently, the spectrometer was coupled to an x-ray streak camera to measure the temporal evolution of $\sim 1 \mathrm{keV}$ x-rays produced from laserheated solid materials. A description of the time-resolved instrument is given along with some preliminary data. The physics describing the heating mechanism of these systems is being investigated using the time-resolved data and radiative hydrodynamic simulations of the experiments.

\section{INSTRUMENT DESCRIPTION}

Figure 1 provides a schematic of the variable-spaced grating "VSG" spectrometer. In these experiments, the VSG measures soft $\mathrm{x}$-ray emission in the spectral range of $8-22 \AA$. The instrument consists of a Perkin-Elmer variable-line spaced grating (modeled after Ref 3,4 ) housed inside an aluminum body. The grating has a nominal groove spacing of 2400 lines $/ \mathrm{mm}$ at the center of a $3 \mathrm{~cm} \times 5 \mathrm{~cm}$ gold-coated Zerodur substrate. The variable spaced grating focuses the $\mathrm{x}$-rays onto a plane instead of the Rowland circle, making the instrument a 'flat-field spectrometer' ${ }^{3-5}$ The instrument was designed to have an axis of

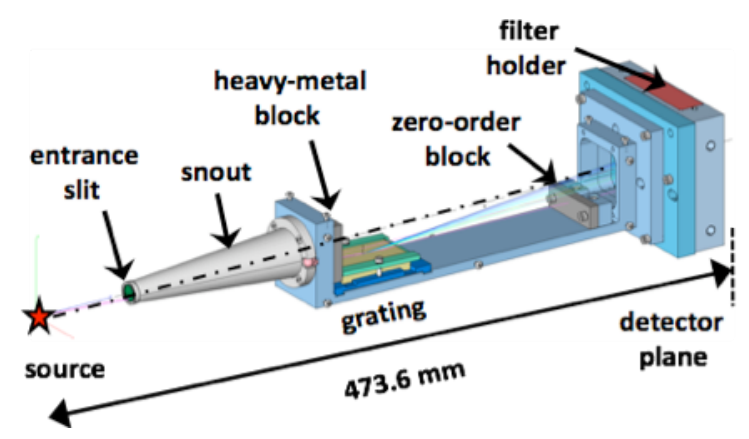

FIG. 1. VSG soft $x$-ray grating spectrometer

rotation around a straight line from the source to the center of the detector plane, allowing the grating plus entrance slit to rotate by $90^{\circ}$ to accommodate different experimental setups. Thus, the grating is secured inside of the aluminum body and tilted at an angle of $1.8^{\circ}$ from the horizontal towards the detector in order to assure an incident angle of $1.3^{\circ}$. The distance from the source to the center of the detector is $473.6 \mathrm{~mm}$. The distance an x-ray travels from the source to the center of the grating is $236 \mathrm{~mm}$. The experimental setup uses the grating on the bottom (relative to the axis-of-rotation) as shown in Figure 1. A horizontal entrance slit $(4.28 \mathrm{~mm}$ wide $\times .09 \mathrm{~mm}$ high) positioned parallel to the grooves of the grating at the front tip of the snout provides a line of sight for x-rays from the target onto the grating. A heavymetal tungsten alloy block at the front of the aluminum body prevents straight-thru x-rays from directly illuminating the detector. A second block is positioned before the detector plane to block the zero-order diffracted x-rays (reflected off the grating) from illuminating the detector. A filter composed of $1600 \AA$ aluminum coated onto $5000 \AA$ of polypropylene $\left(\mathrm{C}_{3} \mathrm{H}_{6}\right)$ 
positioned between the grating and detector reduces the background signal due to scattered optical light within the instrument.

The VSG spectrometer was coupled to a commercially available x-ray streak camera (XRSC) manufactured by Kentech Instruments. The streak camera was outfitted with a vertical slit of width $0.4375 \mathrm{~mm}$ and a photocathode composed of an aluminum photoconductive layer coated onto Lexan $\left(\mathrm{C}_{16} \mathrm{H}_{14} \mathrm{O}_{3}\right)$. The Luxél Corporation fabricated the photocathode. The photocathode was coated with cesium iodide for increased sensitivity to photons at $1 \mathrm{keV}$ - providing Lexan/Al/CsI layers of thickness $949 \AA, 250 \AA$, and $1200 \AA$, respectively. The Kentech streak camera was mated to a $40 \mathrm{~mm}$ diameter Photek microchannel plate image intensifier and a $4096 \times 4096$ pixel array CCD camera from Spectral Instruments. Each CCD pixel has a dimension of $9 \mu \mathrm{m} \times 9 \mu \mathrm{m}$. The Kentech was operated on sweep speed 3, which corresponds to a time resolution of $0.53 \mathrm{ps} / \mathrm{pixel}$ on the CCD camera and a time window of about $\sim 2.1 \mathrm{~ns}$. The internal magnification of the electron optics within the Kentech $\mathrm{XRSC}$ is $\sim 1.185$. With a vertical entrance photocathode slit of $0.4375 \mathrm{~mm}$ and a sweep speed setting of $59 \mathrm{ps} / \mathrm{mm}$ (sweep speed 3 ), the overall time resolution is determined to be $31 \mathrm{ps}$.

\section{EXPERIMENT}

The experiments were performed using the COMET laser at the LLNL Jupiter Laser Facility. COMET is a hybrid chirpedpulse amplification laser consisting of a Ti:sapphire oscillator and a regenerative amplifier tuned to $1053 \mathrm{~nm}$, with a four-stage $\mathrm{Nd}$ :phosphate glass amplifier. ${ }^{6}$ Only one beam of the COMET platform was used for the experiments described in this paper. In these experiments, the laser energy and pulse length varied from 4 to $6 \mathrm{~J}$ on target and between $300-600 \mathrm{ps}$, respectively. COMET utilizes an $f / 3.72$ off-axis parabola to focus the beam to a minimum spot size of $\sim 10 \mu \mathrm{m}$ diameter at full-width-halfmaximum (FWHM). The spot size was changed to deliver intensities ranging from $5 \times 10^{13}-1 \times 10^{16} \mathrm{~W} / \mathrm{cm}^{2}$.

For these experiments, the COMET laser was frequency doubled to a wavelength of $527 \mathrm{~nm}$ in an effort to minimize the prepulse and prevent interaction of the laser with any preformed plasma in front of the target. The temporal pulse shape of the laser was measured with an ultrafast photodiode $(<40 \mathrm{ps}$ risetime) manufactured by Electron Optics Technology. The targets were thin mylar $\left(\mathrm{C}_{10} \mathrm{H}_{8} \mathrm{O}_{4}\right)$ and copper foils irradiated at $8^{\circ}$ from normal. The VSG spectrometer was mounted inside the interaction chamber behind the target at $40^{\circ}$ from normal so that it looked at the back of the target, as shown in Figure 2. The streak camera was mounted behind the VSG spectrometer. The high voltage necessary for operation of the streak camera required a vacuum of $10^{-6}$ torr inside the target chamber.

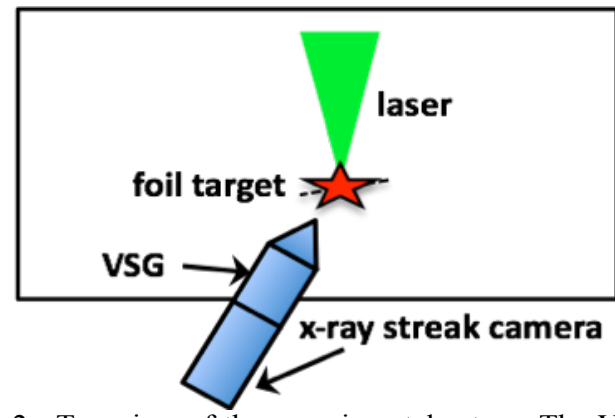

FIG. 2. Top view of the experimental setup. The VSG was looking at the back of the target.

\section{DATA}

The spectral dispersion of the time-resolved VSG spectrometer was calibrated using the spectrum from a $1.5 \mu \mathrm{m}$ thin mylar foil target. The COMET laser delivered $5.5 \mathrm{~J}$ of energy focused to a $10 \mu \mathrm{m}$ diameter spot on the mylar foil. The pulse duration was 415 ps (FWHM), generating a peak intensity of $1.68 \times 10^{16} \mathrm{~W} / \mathrm{cm}^{2}$ on target. Figure $3 \mathrm{a}$ shows unprocessed streaked spectrum measured from the laser-heated mylar foil. Figure $3 \mathrm{~b}$ is a lineout over the entire mylar spectrum, showing several dominant spectral features due to highly charged oxygen ions. The line positions from oxygen ions used to confirm the spectral dispersion of the instrument were: Ly- $\gamma$ at $15.176 \AA$, Ly$\beta$ at $16.006 \AA, \mathrm{He}-\beta$ at $18.627 \AA, \mathrm{Ly}-\alpha$ at $18.967 \AA$, and $\mathrm{He}-\alpha$ at $21.602 \AA^{7-9}$ The spectral resolution $(\lambda / \Delta \lambda)$ of the spectrometer coupled to the streak camera was measured to be 85 at $15.176 \AA$ and 130 at $18.967 \AA$, respectively.

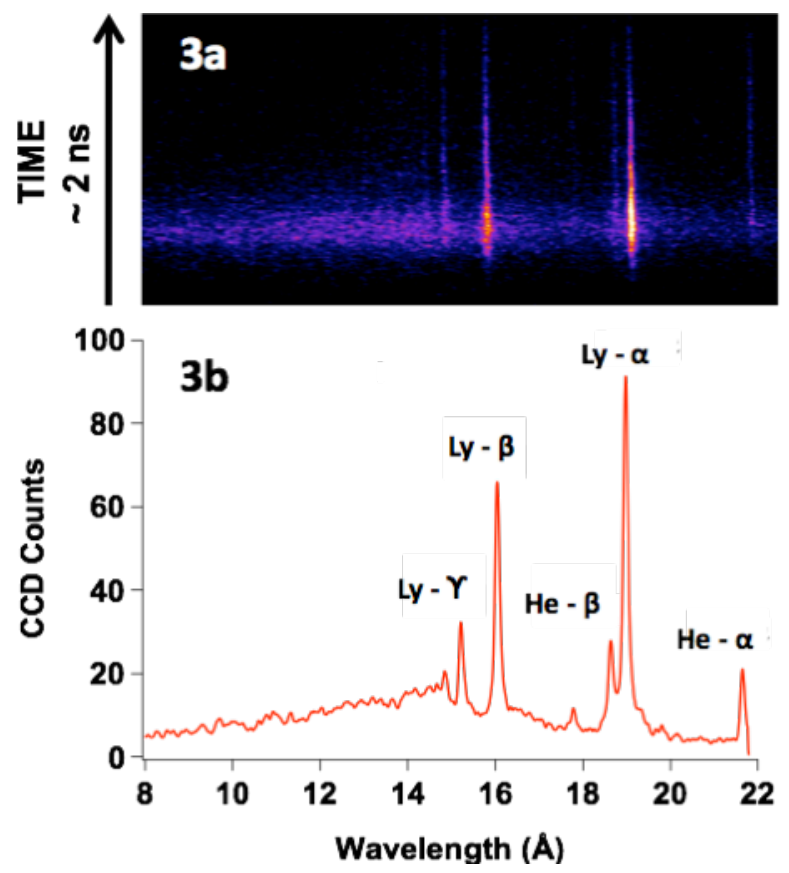

FIG. 3. (a) Unprocessed streaked spectrum from a laser heated mylar foil. (b) Lineout of streaked mylar spectrum over $\sim 2.1 \mathrm{~ns}$ showing prominent H-like and He-like oxygen lines used for the spectral calibration of the spectrometer.

The VSG was used to study the heating mechanism of mid-Z targets as a function of laser intensity. Copper was chosen for the study because it has intense L-shell emission at an x-ray energy of $1 \mathrm{keV}(12.398 \AA)$. Streaked spectra from copper-heated foils were measured to investigate the temporal evolution of x-ray emission under different laser-heating conditions. Figure 4 shows a typical dataset. A 350 ps (FWHM) laser pulse with 4.48 $\mathrm{J}$ of energy was delivered onto a target composed of $9000 \AA$ of copper coated onto $0.5 \mu \mathrm{m}$ of plastic. The laser was focused to a $25 \mu \mathrm{m}$ diameter spot with a peak intensity of $2.61 \times 10^{15} \mathrm{~W} / \mathrm{cm}^{2}$. Figure 4a shows an unprocessed streaked spectrum measured from the back side of the laser-heated copper foil. Figure $4 \mathrm{~b}$ is a lineout in the region of $\mathrm{Cu}$ L-shell emission $(11.2 \AA-12.3 \AA)$ over a $1 \mathrm{~ns}$ time window. Figure $4 \mathrm{c}$ shows the photodiode trace of the laser pulse. The relative timing between the soft $\mathrm{x}$-ray and photodiode signals were adjusted so that $800 \mathrm{ps}$ corresponds to the peak of each signal. 

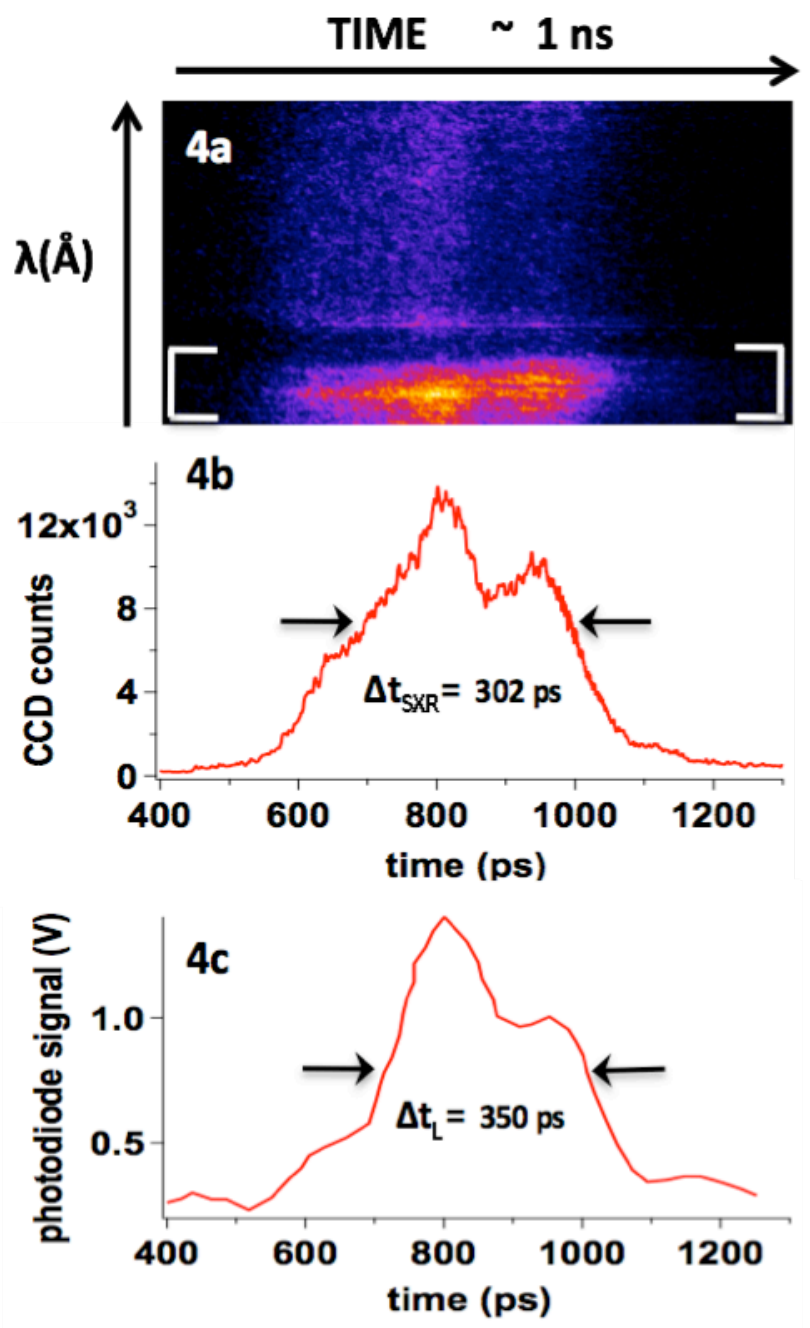

FIG. 4. (a) Unprocessed streaked spectrum from a $\mathrm{Cu}$ foil heated by a $2.61 \times 10^{15} \mathrm{~W} \mathrm{~cm}^{-2}$ laser pulse delivered in 350 ps. The horizontal lineout reproduced in part $b$ is the region enclosed by white brackets. (b) Lineout through streaked spectrum of laser-heated $\mathrm{Cu}$ foil, showing temporal evolution of $\sim 1 \mathrm{keV}$ x-ray emission $(11.2 \AA-12.3 \AA)$. (c) Photodiode monitor of the COMET laser pulse

\section{DISCUSSION}

The photodiode monitoring the temporal shape of the COMET laser pulse measured structure in the intensity of the laser over time (Figure 4c). The time-resolved spectrum measured from the back side of a copper foil also showed structure in the intensity of x-rays emitted over time (Figure 4b), indicating that the soft $\mathrm{x}$-ray emission follows the laser heating of the target. Temporal measurements of the laser pulse and copper L-shell emission in an x-ray band around $1 \mathrm{keV}$ were similar in duration: 350 ps and 302 ps at FWHM, respectively.
The VSG spectrometer was developed to study the heating mechanisms of thin foil targets irradiated by short-pulse lasers. Data will be analyzed to determine temperature and density conditions of the plasma with the help of the radiationhydrodynamics code, HYDRA. ${ }^{10,11}$ The grating sensitivity has been calibrated using the Electron Beam Ion Trap (EBIT-I) at LLNL. $^{12}$ Data analysis of the entire study is fairly detailed and will be published elsewhere.

A reflection grating spectrometer has been coupled to a Kentech XRSC. The spectral resolution of the instrument is comparable to others previously developed $(\lambda / \Delta \lambda \sim 100) .{ }^{13,14}$ The new compact design of the spectrometer allows rotation of the grating plus entrance slit by $90^{\circ}$ increments for use with different experiment geometries of each laser platform available within LLNL's Jupiter Laser Facility. Furthermore, the time resolution of the diagnostic may be improved from 31 ps to 3 ps by using the highest sweep speed setting available on the Kentech streak camera. Several experiments were carried out to study the temporal $\mathrm{x}$-ray emission of targets irradiated under different conditions using the COMET laser. This diagnostic is a crucial tool to study $\mathrm{x}$-ray emission from short-pulse laser-produced plasmas.

\section{ACKNOWLEDGEMENTS}

We would like to acknowledge technical support from Ed Magee, Dwight Price, Jim Hunter, Rob Costa, Chuck Cadwalader, and the JLF operations staff. This work was performed under the auspices of the U.S. Department of Energy by the Lawrence Livermore National Laboratory under Contract DE-AC52-07NA27344 and LDRD 08-ERD-024. K. V. Cone is a Gates Millennium Scholar supported by the Bill and Melinda Gates Foundation.

${ }^{1}$ H. R. Griem, Phys. Fluids B. 4, 2436 (1992).

${ }^{2}$ M. B. Schneider, D. E. Hinkel, S. J. Moon, S. B Hansen, et al., High Energy Density Phys. 3, 256 (2007).

${ }^{3}$ T. Harada and T. Kita, Appl. Opt. 19, 3987 (1980).

${ }^{4}$ N. Nakano, H. Kuroda, T. Kita, and T. Harada, Appl. Opt. 23, 2386 (1984).

${ }^{5}$ J. L. Schwob, A. W. Wouters, S. Suckewer, and M. Finkenthal, Rev. Sci. Instrum. 58, 1601 (1987).

${ }^{6}$ J. Dunn, J. Nilsen, A. L. Osterheld, and V. Shlyaptsev, Opt. Lett. 24, 101 (1999).

${ }^{7}$ G. W. Drake, Can. J. Phys. 66, 586 (1988).

${ }^{8}$ J. D. Garcia and J. E. Mack, J. Opt. Soc. Am. 55, 654 (1965).

${ }^{9}$ L. A. Vainshtein and U. I. Safranova, Phys. Scr. 31, 519 (1985).

${ }^{10}$ M. M. Marinak, S. W. Haan, T. R. Dittrich, R. E. Tipton, and G. B. Zimmerman, Phys. Plasmas 5, 1125 (1998).

${ }^{11}$ M. M. Marinak, G. D. Kerbel, N. A. Gentile, O. Jones, D. Munro, S. Pollaine, T. R. Dittrich, and S. W. Haan, Phys. Plasmas 8, 2275 (2001).

${ }^{12}$ J. Park, G. V. Brown, M. B. Schneider, H. A. Baldis, et al., Rev. Sci. Instrum. (in these proceedings).

${ }^{13}$ R. T. Eagleton and S. F. James. Rev. Sci. Instrum. 75, 3969 (2004).

${ }^{14}$ G. D. Tsakiris and R. Sigel. Phys. Rev. A 38, 5769 (1988). 